Скопје, Македонија

\title{
A STUDY OF FUNCTOR ASSOCIATED WITH TRANSFORMATION GROUPS
}

\author{
PRAVANJAN KR. RANA
}

\begin{abstract}
The aim of this paper is to construct a functor associated with transformation groups as well as investigate this functor.

In this paper we show that:-

i) for a given transformation group $(X, G)$, where $X$ is a path connected pointed topological space with base point $x_{0}$ and $\mathrm{G}$ is a group of homeomorphisms of $\mathrm{X}$, there always exists a covariant functor ' $\mathbf{F}^{\prime}$ from 'Tgh' to 'Fgh', where 'Tgh' denotes the category of transformation groups and their continuous group homomorphisms and 'Fgh' denotes the category of fundamental groups and their group homomorphisms;

ii) if the transformation groups $(\mathrm{X}, \mathrm{G})$ and $(\mathrm{Y}, \mathrm{H})$ have the same homotopy type, then the groups $\mathrm{F}(\mathrm{X}, \mathrm{G})$ and $\mathrm{F}(\mathrm{Y}, \mathrm{H})$ are isomorphic; we also prove that

iii) The covariant functor $F: \mathbf{T g h} \rightarrow \mathbf{F g h}$ is a homotopy type invariant.
\end{abstract}

\section{Introduction}

Throught this paper we assume that $\mathrm{X}$ is a path connected pointed topological space with base point $x_{0}$. For simplicity, we write $(X, G)$ in place of $\left(\mathrm{X}, x_{0}, \mathrm{G}\right)$.

Now we recall the following definitions and statements:-

\section{Definiton 1.1}

A transformation group is a pair $(\mathrm{X}, \mathrm{G})$, where $\mathrm{X}$ is a path connected pointed topological space with base point $x_{0}$ and $\mathrm{G}$ is a group of homeomorphisms of X.

A map $(\Phi, \Psi):(X, G) \longrightarrow(Y, H)$ consists of a continuous map $\Phi$ : $X \longrightarrow Y$ and a homomorphism $\Psi: G \longrightarrow H$, such that

$$
\Phi(g x)=\Psi(g) \Phi(x), \text { for every pair }(x, g) .
$$

\section{Definiton 1.2}

Given any element $\mathrm{g}$ of $\mathrm{G}$, a path $\alpha$ of order $\mathrm{g}$ with base point $x_{0}$ is a continuous map $\alpha: I \longrightarrow X$ such that $\alpha(0)=x_{0}, \alpha(1)=g x_{0}$.

2000 Mathematics Subject Classification. 55U40, 55P65.

Key words and phrases. Category, Covariant functor, Same homotopy type. 
A path $\alpha$ of order $g_{1}$ and a path $\beta$ of order $g_{2}$ form a new path $\alpha+g_{1} \beta$ of order $g_{1} g_{2}$ defined by the following equations:

$\left(\alpha+g_{1} \beta\right)(t)=\alpha(2 t), 0 \leq t \leq 1 / 2$

$\left(\alpha+g_{1} \beta\right)(t)=g_{1} \beta(2 t-1), 1 / 2 \leq t \leq 1$.

Two paths $\alpha$ and $\beta$ of same order g are said to be homotopic iff $\exists$ a continuous map

$C: I \times I \rightarrow X$ such that,

$C(s, 0)=\alpha(s), 0 \leq s \leq 1$

$C(s, 1)=\beta(s), 0 \leq s \leq 1$

$C(0, t)=x_{0}, 0 \leq t \leq 1$

$C(1, t)=g x_{0}, 0 \leq t \leq 1$.

Let $[\alpha ; g]$ denotes the homotopy class of a path $\alpha$ of the order g. The family of all such homotopy classes of paths of prescribed order with the rule of composition ' $\diamond$ ' is a group, where ' $\diamond$ ' is defined by

$\left[\alpha ; g_{1}\right] \diamond\left[\beta ; g_{2}\right]=\left[\alpha+g_{1} \beta ; g_{1} g_{2}\right]$

This group is called the fundamental group of $(\mathrm{X}, \mathrm{G})$ with base point $x_{0}$ and is denoted by $\mathbf{F}(\mathrm{X}, \mathrm{G})$.

Definition 1.3

A category $\mathbf{C}$ consists of

(a) a class of objects $\mathrm{X}, \mathrm{Y}, \mathrm{Z}, \ldots$, denoted by $\mathrm{Ob}(\mathbf{C})$;

(b) for each ordered pair of objects $\mathrm{X}, \mathrm{Y}$ a set of morphisms with domain $\mathrm{X}$ and range $\mathrm{Y}$ denoted by $\mathbf{C}(\mathrm{X}, \mathrm{Y})$;

(c) for each order triple of objects $\mathrm{X}, \mathrm{Y}$ and $\mathrm{Z}$ and a pair of morphisms

$\mathrm{f}: \mathrm{X} \longrightarrow Y$ and $\mathrm{g}: \mathrm{Y} \longrightarrow \mathrm{Z}$ their composite is denoted by $\mathrm{gf}: \mathrm{X} \longrightarrow \mathrm{Z}$, satisfying the following two axioms:

i) associativity : if $f \in \mathbf{C}(\mathrm{X}, \mathrm{Y}), g \in \mathbf{C}(\mathrm{Y}, \mathrm{Z})$ and $h \in \mathbf{C}(\mathrm{Z}, \mathrm{W})$, then

$h(g f)=(h g) f \in \mathbf{C}(\mathrm{X}, \mathrm{W})$.

ii) identity : for each object $\mathrm{Y}$ in $\mathbf{C}$ there is a morphism $I_{Y} \in \mathbf{C}(\mathrm{Y}, \mathrm{Y})$ such that if $f \in \mathbf{C}(\mathbf{X}, \mathbf{Y})$ then $I_{Y} f=f$ and if $h \in \mathbf{C}(\mathrm{Y}, \mathrm{Z})$, then $\mathrm{h} I_{Y}=\mathrm{h}$.

Definition 1.4

Let $\mathbf{C}$ and $\mathbf{D}$ be categories. A covariant functor $\mathrm{T}$ from $\mathbf{C}$ to $\mathbf{D}$ consists of i) an object function which assigns to every object $\mathrm{X}$ of $\mathbf{C}$ an object $\mathrm{T}(\mathrm{X})$ of $\mathbf{D}$; and

ii) a morphism function which assigns to every morphism $\mathrm{f}: \mathrm{X} \longrightarrow \mathrm{Y}$ in $\mathbf{C}$, a morphism $T(f): T(X) \longrightarrow T(Y)$ in $\mathbf{D}$ such that

a) $T\left(I_{X}\right)=I_{T(X)}$;

b) $T(g f)=T(g) \cdot T(f)$, for $\mathrm{g}: \mathrm{Y} \longrightarrow \mathrm{W}$ in $\mathbf{C}$. 


\section{Definition 1.5}

Two transformation groups $(\mathrm{X}, \mathrm{G})$ and $(\mathrm{Y}, \mathrm{H})$ will said to be of the same homotopy type if there exists category mappings

$(\Phi, \Psi):(X, G) \longrightarrow(Y, H)$ and $\left(\Phi^{\prime}, \Psi^{\prime}\right):(Y, H) \longrightarrow(X, G)$,

where $\Psi$ and $\Psi^{\prime}$ are isomorphisms and $\Phi^{\prime} \Phi \simeq I_{X}$ and $\Phi \Phi^{\prime} \simeq I_{Y}$.

\section{Lemma 1.6}

The transformation groups and its continuous group homomorphisms forms a category.

Proof:- We take all transformation groups $(\mathrm{X}, \mathrm{G})$ as the set of objects and the set of all transformation groups homomorphisms, the set of morphisms and the composition is the usual composition of mappings. This category will be denoted by 'Tgh'.

\section{Lemma 1.7}

Let $(\mathrm{X}, \mathrm{G})$ and $(\mathrm{Y}, \mathrm{H})$ be transformation groups. If $f_{1}$ and $f_{2}$ are two homotopic paths in X with base point $x_{0}$ of order $g_{1}$ and $g_{2}$ respectively, then $h_{1} f_{1}$ and $h_{1} f_{2}$ are also two homotopic paths in $\mathrm{Y}$ with base point $y_{0}$ of order $h_{2} g_{1}$ and $h_{2} g_{2}$ respectively, where $g_{1}, g_{2} \in G$ and $\left(h_{1}, h_{2}\right):(X, G) \rightarrow(Y, H)$ be a continuous group homomorphism , i.e; $\left[f_{1} ; g_{1}\right]=\left[f_{2} ; g_{2}\right] \Longrightarrow\left[h_{1} f_{1} ; h_{2} g_{1}\right]=\left[h_{1} f_{2} ; h_{2} g_{2}\right]$.

Proof:- Using Definition1.1 and Definition1.2, it follows.

In Section 2, we construct and investigate functor associated with transformation groups.

2) Functor associated with transformation groups.

We now construct functor associated with transformation groups.

Let $(\mathrm{X}, \mathrm{G})$ be a transformation group, where $\mathrm{X}$ is a path connected pointed topological space with base point $x_{0}$ and $\mathrm{G}$ is a group of homeomorphisms of $\mathrm{X}$,

Then we have the following Theorems:-

Theorem 2.1:-

Let $I_{(X, G)}:(X, G) \longrightarrow(\mathrm{X}, \mathrm{G})$ be a mapping,then $\left(I_{(X, G)}\right)_{*}: F(X, G) \longrightarrow \mathrm{F}(\mathrm{X}, \mathrm{G})$ is a group homomorphism.

Proof:- Define $\left(I_{(X, G)}\right)_{*}: F(X, G) \longrightarrow \mathrm{F}(\mathrm{X}, \mathrm{G})$ by $\left(I_{(X, G)}\right)_{*}([\alpha ; g])=\left[I_{X}(\alpha) ; I_{G}(g)\right]=[\alpha ; g]$.

Let $\left[\alpha_{1} ; g_{1}\right],\left[\alpha_{2} ; g_{2}\right] \in \mathrm{F}(\mathrm{X}, \mathrm{G})$.

Then $\left[\alpha_{1} ; g_{1}\right]=\left[\alpha_{2} ; g_{2}\right]$

$\Rightarrow\left[I_{X}\left(\alpha_{1}\right) ; I_{G}\left(g_{1}\right)\right]=\left[I_{X}\left(\alpha_{2}\right) ; I_{G}\left(g_{2}\right)\right]$

$\Rightarrow\left(I_{(X, G)}\right)_{*}$ is well defined.

Now $\left(I_{(X, G)}\right)_{*}\left(\left[\alpha_{1} ; g_{1}\right] \diamond\left[\alpha_{2} ; g_{2}\right]\right)=\left(I_{(X, G)}\right)_{*}\left(\left[\alpha_{1}+g_{1} \alpha_{2} ; g_{1} g_{2}\right]\right)$.

Thus $\left(I_{(X, G)}\right)_{*}\left(\left[\alpha_{1}+g_{1} \alpha_{2} ; g_{1} g_{2}\right]\right)$

$=\left[I_{X}\left(\alpha_{1}+g_{1} \alpha_{2}\right) ; I_{G}\left(g_{1} g_{2}\right)\right]$ 
$=\left[\alpha_{1}+g_{1} \alpha_{2} ; g_{1} g_{2}\right]$

$=\left[\alpha_{1} ; g_{1}\right] \diamond\left[\alpha_{2} ; g_{2}\right]$

$=\left(I_{(X, G)}\right) *\left(\left[\alpha_{1} ; g_{1}\right]\right) \diamond\left(I_{(X, G)}\right)_{*}\left(\left[\alpha_{2} ; g_{2}\right]\right)$

Hence $\left(I_{(X, G)}\right)_{*}\left(\left[\alpha_{1} ; g_{1}\right] \diamond\left[\alpha_{2} ; g_{2}\right]\right)=\left(I_{(X, G)}\right)_{*}\left(\left[\alpha_{1} ; g_{1}\right]\right) \diamond\left(I_{(X, G)}\right)_{*}\left(\left[\alpha_{2} ; g_{2}\right]\right)$.

Theorem 2.2:-

Let $\alpha:(X, G) \longrightarrow(\mathrm{Y}, \mathrm{G})$ be a category mapping between two transformation groups $(\mathrm{X}, \mathrm{G})$ and $(\mathrm{Y}, \mathrm{G})$, then $\alpha_{*}: F(X, G) \longrightarrow \mathrm{F}(\mathrm{Y}, \mathrm{G})$ is a group homomorphism .

Proof:- Let $\alpha_{*}: F(X, G) \rightarrow \mathrm{F}(\mathrm{Y}, \mathrm{G})$ be defined by $\alpha_{*}([f ; g])=[\alpha f ; g], \forall g \in G$.

Next let $\left[f_{1} ; g_{1}\right],\left[f_{2} ; g_{2}\right] \in \mathrm{F}(\mathrm{X}, \mathrm{G})$.

If $\left[f_{1} ; g_{1}\right]=\left[f_{2} ; g_{2}\right]$

$\Rightarrow\left[\alpha f_{1} ; g_{1}\right]=\left[\alpha f_{2} ; g_{1}\right]$

$\Rightarrow \alpha_{*}\left(\left[f_{1} ; g_{1}\right]\right)=\alpha_{*}\left[f_{2}: g_{1}\right]$

$\Rightarrow \alpha_{*}$ is well defined.

Next let $\left[\alpha_{1} ; g_{1}\right],\left[\alpha_{2} ; g_{2}\right] \in \mathrm{F}(\mathrm{X}, \mathrm{G})$.

Then $\alpha_{*}\left(\left[\alpha_{1} ; g_{1}\right] \diamond\left[\alpha_{2} ; g_{2}\right]\right)=\alpha_{*}\left(\left[\alpha_{1}+g_{1} \alpha_{2} ; g_{1} g_{2}\right]\right), \forall g_{1}, g_{2} \in G$

Now $\alpha_{*}\left(\left[\alpha_{1}+g_{1} \alpha_{2} ; g_{1} g_{2}\right]\right)=\left[\alpha\left(\alpha_{1}+g_{1} \alpha_{2}\right) ; g_{1} g_{2}\right]$

$=\left[\alpha \alpha_{1} ; g_{1}\right] \diamond\left[\alpha \alpha_{2} ; g_{2}\right]=\alpha_{*}\left(\left[\alpha_{1} ; g_{1}\right]\right) \diamond \alpha_{*}\left(\left[\alpha_{2} ; g_{2}\right]\right)$.

Thus $\alpha_{*}: F(X, G) \longrightarrow \mathrm{F}(\mathrm{Y}, \mathrm{G})$ is a group homomorphism .

Theorem 2.3:-

Let $\left(h_{1}, h_{2}\right):(X, G) \longrightarrow(\mathrm{Y}, \mathrm{H})$ be a category mapping between two transformation groups $(\mathrm{X}, \mathrm{G})$ and $(\mathrm{Y}, \mathrm{H})$, then $\left(h_{1}, h_{2}\right)_{*}: F(X, G) \longrightarrow \mathrm{F}(\mathrm{Y}, \mathrm{H})$ is a group homomorphism .

Proof:- Define $\left(h_{1}, h_{2}\right)_{*}: F(X, G) \longrightarrow \mathrm{F}(\mathrm{Y}, \mathrm{H})$ by $\left(h_{1}, h_{2}\right)_{*}([f ; g])=\left[h_{1} f ; h_{2} g\right]$.

Let $\left[f_{1} ; g_{1}\right],\left[f_{2} ; g_{2}\right] \in \mathrm{F}(\mathrm{X}, \mathrm{G})$.

If $\left[f_{1} ; g_{1}\right]=\left[f_{2} ; g_{2}\right]$

$\Rightarrow\left[h_{1} f_{1} ; h_{2} g_{1}\right]=\left[h_{1} f_{2} ; h_{2} g_{2}\right]$, by lemma 1.7 .

Thus $\left[f_{1} ; g_{1}\right]=\left[f_{2} ; g_{2}\right]$

$\Rightarrow\left(h_{1}, h_{2}\right)_{*}\left(\left[f_{1} ; g_{1}\right]\right)=\left(h_{1}, h_{2}\right)_{*}\left(\left[f_{2} ; g_{2}\right]\right)$.

$\Rightarrow$ This map is well defined.

Now $\left(h_{1}, h_{2}\right)_{*}\left(\left[f_{1} ; g_{1}\right] \diamond\left[f_{2} ; g_{2}\right]\right)$

$=\left(h_{1}, h_{2}\right)_{*}\left(\left[f_{1}+g_{1} f_{2} ; g_{1} g_{2}\right]\right)$

$\left.=\left[h_{1}\left(f_{1}+g_{1} f_{2}\right) ; h_{2}\left(g_{1} g_{2}\right)\right]\right)$.

$=\left[h_{1} f_{1}+h_{1}\left(g_{1} f_{2}\right) ; h_{2}\left(g_{1}\right) h_{2}\left(g_{2}\right)\right]$

$=\left[h_{1} f_{1}+\left(h_{2} g_{1}\right)\left(h_{1} f_{2}\right) ; h_{2}\left(g_{1}\right) h_{2}\left(g_{2}\right)\right]$

$=\left[h_{1} f_{1} ; h_{2} g_{1}\right] \diamond\left[h_{1} f_{2} ; h_{2} g_{2}\right]$,

$=\left(h_{1}, h_{2}\right)_{*}\left(\left[f_{1} ; g_{1}\right] \diamond\left(h_{1}, h_{2}\right)_{*}\left(\left[f_{2} ; g_{2}\right]\right)\right.$

Thus $\left(h_{1}, h_{2}\right)_{*}: F(X, G) \longrightarrow \mathrm{F}(\mathrm{Y}, \mathrm{H})$ is a group homomorphism . 


\section{Proposition 2.4:-}

Let $\alpha:(X, G) \rightarrow(X, H)$ be a category mapping, $\alpha$ induces a group homomorphism, $\alpha_{*}: F(X, G) \rightarrow F(X, H)$, where $\alpha_{*}=\left(I_{X}, \alpha\right)_{*}$.

Proof:- Let $[f ; g] \in F(X, G)$. We define $\alpha_{*}: F(X, G) \rightarrow F(X, H)$ by $\alpha_{*}([f ; g])=[f ; \alpha g]$.

Let $\left[f_{1} ; g_{1}\right]=\left[f_{2} ; g_{2}\right] \Rightarrow\left[f_{1} ; \alpha g_{1}\right]=\left[f_{2} ; \alpha g_{2}\right] \Rightarrow \alpha_{*}\left(\left[f_{1} ; g_{1}\right]\right)=\alpha_{*}\left(\left[f_{2} ; g_{2}\right)\right.$.

$\Rightarrow \alpha_{*}$ is well defined.

Now $\alpha_{*}\left(\left[f_{1} ; g_{1}\right] \diamond\left[f_{2} ; g_{2}\right]\right)$

$=\alpha_{*}\left(\left[f_{1}+g_{1} f_{2} ; g_{1} g_{2}\right]\right)$

$=\left[f_{1}+g_{1} f_{2} ; \alpha\left(g_{1} g_{2}\right)\right]$

$=\left[f_{1}+\left(\alpha g_{1}\right) f_{2} ;\left(\alpha g_{1}\right)\left(\alpha g_{2}\right)\right]$

$=\left[f_{1} ; \alpha g_{1}\right] \diamond\left[f_{2} ; \alpha g_{2}\right]$.

Thus $\alpha_{*}\left(\left[f_{1} ; g_{1}\right] \diamond\left[f_{2} ; g_{2}\right]\right)=\alpha_{*}\left(\left[f_{1} ; g_{1}\right]\right) \diamond \alpha\left(\left[f_{2} ; g_{2}\right]\right)$.

$\Rightarrow \alpha_{*}$ is a group homomorphism.

Theorem 2.5:-

Let ' $\mathbf{T g h}$ ' denotes the category of transformation groups and their continuous group homomorphisms and 'Fgh' denotes the category of fundamental groups and their group homomorphisms.

Then $F: \mathbf{T g h} \rightarrow \mathbf{F g h}$ is a covariant functor.

Proof:- Let (X,G) be a transformation group in 'Tgh', then F(X,G) is a fundamental group in ' $\mathbf{F g h}$ '.

Also for $\left(h_{1}, h_{2}\right):(X, G) \rightarrow(Y, H)$ in 'Tgh' $\left(h_{1}, h_{2}\right)_{*}: F(X, G) \rightarrow F(Y, H)$ in 'Fgh' by $\left(h_{1}, h_{2}\right)_{*}([f ; g])=\left[h_{1} f ; h_{2} g\right]$.

Let $\left(\alpha_{1}, \alpha_{2}\right):(X, G) \rightarrow(Y, H),\left(\beta_{1}, \beta_{2}\right):(Y, H) \rightarrow(Z, W)$ are continuous group homomorphism, then $\left(\beta_{1}, \beta_{2}\right) \cdot\left(\alpha_{1}, \alpha_{2}\right)=\left(\beta_{1} \alpha_{1}, \beta_{2} \alpha_{2}\right):(X, G) \rightarrow$ $(Z, W)$ is also a continuous group homomorphism.

Thus $\left(\beta_{1} \alpha_{1}, \beta_{2} \alpha_{2}\right)_{*}: F(X, G) \rightarrow F(Z, W)$ by

$\left(\beta_{1} \alpha_{1}, \beta_{2} \alpha_{2}\right)_{*}([f ; g])=\left[\left(\beta_{1} \alpha_{1}\right) f ;\left(\beta_{2} \alpha_{2}\right) g\right]$

$=\left[\beta_{1}\left(\alpha_{1} f\right) ; \beta_{2}\left(\alpha_{2} g\right)\right]=\left(\beta_{1}, \beta_{2}\right)_{*}\left[\alpha_{1} f ; \alpha_{2} g\right]=\left(\beta_{1}, \beta_{2}\right)_{*}\left(\alpha_{1}, \alpha_{2}\right)_{*}$

Thus $\left(\left(\beta_{1}, \beta_{2}\right) \cdot\left(\alpha_{1}, \alpha_{2}\right)\right)_{*}=\left(\beta_{1}, \beta_{2}\right)_{*}\left(\alpha_{1} \alpha_{2}\right)_{*}$.

Also, for $I_{(X, G)}:(X, G) \rightarrow(X, G)$,

$F\left(I_{(X, G)}\right): F(X, G) \rightarrow F(X, G)$ by

$F\left(I_{(X, G)}\right)([f ; g])=[f ; g]=I_{F(X, G)}$

$\Rightarrow$ ' $\mathrm{F}$ ' is a covariant functor.

Theorem 2.6:-

If $(\mathrm{X}, \mathrm{G})$ and $(\mathrm{Y}, \mathrm{H})$ be two transformation groups having same homotopy type, then the groups $\mathrm{F}(\mathrm{X}, \mathrm{G})$ and $\mathrm{F}(\mathrm{Y}, \mathrm{H})$ are isomorphic, where $G \simeq H$ and $X \simeq Y$.

Proof:- Since $(\mathrm{X}, \mathrm{G})$ and $(\mathrm{Y}, \mathrm{H})$ have the same homotopy type, then there exists 
$\left(\alpha_{1}, \alpha_{2}\right):(X, G) \rightarrow(Y, H)$ and $\left(\beta_{1}, \beta_{2}\right):(Y, H) \rightarrow(X, G)$ such that $\alpha_{1} \circ \beta_{1} \simeq I_{Y}$ and $\beta_{1} \circ \alpha_{1} \simeq I_{X}$ and $\alpha_{2} \circ \beta_{2} \simeq I_{H}$ and $\beta_{2} \circ \alpha_{2} \simeq I_{G}$.

Let $\left(\alpha_{1}, \alpha_{2}\right)_{*}: F(X, G) \rightarrow F(Y, H)$ be defined by

$\left(\alpha_{1}, \alpha_{2}\right)_{*}([f ; g])=\left[\alpha_{1} f ; \alpha_{2} g\right]$.

Using Theorem2.3 and Lemma1.7, $\left(\alpha_{1}, \alpha_{2}\right)_{*}$ is a homomorphism from $\mathrm{F}(\mathrm{X}, \mathrm{G})$ to $\mathrm{F}(\mathrm{Y}, \mathrm{H})$.

Then $\left(\alpha_{1}, \alpha_{2}\right)_{*}$ satisfies the following properties:-

i) $\left(\alpha_{1}, \alpha_{2}\right) \simeq\left(\beta_{1}, \beta_{2}\right) \Rightarrow\left(\alpha_{1}, \alpha_{2}\right)_{*}=\left(\beta_{1}, \beta_{2}\right)_{*}$

ii) $I_{(X, G)}:(X, G) \rightarrow(X, G)$

$\Rightarrow\left(I_{(X, G)}\right)_{*}=I d \cdot F(X, G)$. $=I d$.

iii) $\left(\left(\beta_{1}, \beta_{2}\right) \cdot\left(\alpha_{1}, \alpha_{2}\right)\right)_{*}=\left(\beta_{1}, \beta_{2}\right)_{*}\left(\alpha_{1}, \alpha_{2}\right)_{*}$.

Since $\alpha_{1} \circ \beta_{1} \simeq I_{Y}$ and $\beta_{1} \circ \alpha_{1} \simeq I_{X}$ and $\alpha_{2} \circ \beta_{2} \simeq I_{H}$ and $\beta_{2} \circ \alpha_{2} \simeq I_{G}$, Thus $\left(\beta_{1} \circ \alpha_{1}, \beta_{2} \circ \alpha_{2}\right) \simeq\left(I_{X}, I_{G}\right)$, by i) and ii) we have

$\left(\beta_{1} \circ \alpha_{1}, \beta_{2} \circ \alpha_{2}\right)_{*}=\left(I_{X}, I_{G}\right)_{*}$.

Hence $\left(\left(\beta_{1}, \beta_{2}\right) \circ\left(\alpha_{1}, \alpha_{2}\right)\right)_{*}=\left(I_{(X, G)}\right)_{*}=I d_{\cdot(F(X, G))}=I d$. , by ii).

Now by iii) we have $\left(\beta_{1}, \beta_{2}\right)_{*}\left(\alpha_{1}, \alpha_{2}\right)_{*}=I d$..

Since $\left(\alpha_{1}, \alpha_{2}\right)_{*}$ is a homomorphism and since $\left(\beta_{1}, \beta_{2}\right)_{*}\left(\alpha_{1}, \alpha_{2}\right)_{*}=I d$. and hence $\left(\alpha_{1}, \alpha_{2}\right)_{*}$ is a monomorphism.

Similarly we show that $\left(\alpha_{1}, \alpha_{2}\right)_{*}\left(\beta_{1}, \beta_{2}\right)_{*}=\mathrm{Id}$. and hence $\left(\alpha_{1}, \alpha_{2}\right)_{*}$ is a epimorphism.

Therefore $\left(\alpha_{1}, \alpha_{2}\right)_{*}$ is an isomorphism .

Thus $\mathrm{F}(\mathrm{X}, \mathrm{G}) \cong \mathrm{F}(\mathrm{Y}, \mathrm{H})$.

Theorem 2.7:-

If two transformation groups $(\mathrm{X}, \mathrm{G})$ and $(\mathrm{X}, \mathrm{H})$ have the same homotopy type, then

$\mathrm{F}(\mathrm{X}, \mathrm{G}) \cong \mathrm{F}(\mathrm{X}, \mathrm{H})$.

Proof:-

Since $(\mathrm{X}, \mathrm{G}) \simeq(\mathrm{X}, \mathrm{H}), \exists \alpha:(X, G) \rightarrow(X, H)$ and $\beta:(X, H) \rightarrow(X, G)$ such that $\alpha \cdot \beta \simeq I_{H}$ and $\beta \cdot \alpha \simeq I_{G}$.

Let $\alpha_{*}: F(X, G) \rightarrow F(X, H)$ be defined by $\alpha_{*}([f ; g])=[f ; \alpha g]$. Using Theorem2.4; $\alpha_{*}$ is a homomorphism.

Then $\alpha_{*}$ satiesfies the following properties:-

i) $\alpha \simeq \beta \Rightarrow \alpha_{*}=\beta_{*}$

ii) $I_{(X, G)}:(X, G) \rightarrow(X, G)$

$\Rightarrow\left(I_{(X, G)}\right)_{*}=I d \cdot F(X, G) .=I d$.

iii) $(\alpha \cdot \beta)_{*}=\alpha_{*} \cdot \beta_{*}$

Since $\beta \cdot \alpha \simeq I_{G}$, by i,ii,iii, we have $(\beta \cdot \alpha)_{*}=\left(I_{G}\right)_{*}$

$\Rightarrow \beta_{*} \cdot \alpha_{*}=I d$.

$\Rightarrow \alpha_{*}$ is a monomorphism. When $\alpha \cdot \beta \simeq I_{H}$, similarly prove that $\alpha_{*}$ is a epimorphism.

Thus $\alpha_{*}$ is an isomorphism and hence the theorem is proved. 
Next we have:-

Theorem 2.8:-

The covariant functor $F: \mathbf{T g h} \rightarrow \mathbf{F g h}$ is a homotopy type invariant.

Proof:- Using the Theorem2.5, it follows that $\mathrm{F}$ is a covariant functor and using the Theorem2.6, it follows that $\mathrm{F}$ is a homotopy type invariant, in the sense that if $(\mathrm{X}, \mathrm{G})$ and $(\mathrm{Y}, \mathrm{H})$ are same homotopy type, then the groups $\mathrm{F}(\mathrm{X}, \mathrm{G})$ and $\mathrm{F}(\mathrm{Y}, \mathrm{H})$ are isomorphic.

\section{REFERENCES}

[1] Rhodes,F:- On the fundamental group of a transformation group.

[2] Adhikary, A. and Rana, P.K. (2001):- A study of functors associated with Topological groups, Studia Univ. "Babes-Bolyai," Mathematica, Vol XLVI, No 4, Dec, 2001.

[3] Rana, P.K.(2009):-A study of the group of covering transformation through functors, BULLETIN MATHEMATIQUE,33(LIX)2009(21-24);

[4] Rana,P.K.(2011):-Astudy of functors associated with rings on continuous function JIAM 32(1),2011(73-78). 


\title{
СТУДИЈА ЗА ФУНКТОР АСОЦИРАН СО ГРУПАТА ОД ТРАНСФОРМАЦИИ
}

\author{
Правањан Кр. Рана
}

Р е $з$ и м е

Целта на овој труд е да се конструира функтор асоциран со групата од трансформации како и да се испита тој функтор. Ќе докажеме дека:

$1)$ за дадена група од трансформации $(X, G)$ каде $\left(X, x_{0}\right)$ е пат сврзан тополошки простор и $G$ е група од хомеоморфизми на $X$, сегогаш постои коваријантен функтор 'F' фром 'Tgh' од 'Tgh' во 'Fgh', каде 'Tgh' ја озна;ува категоријата од групи од трансформации и нејзината непрекината група од хомомотфизми и 'Fgh' ја означува категоријата од фундаментални групи и нивните групи од хомоморфизми.

$2)$ Ако групите од трансформации $(X, G)$ и $(Y, H)$ имаат ист хомотопски тип, тогаш групите $F(X, G)$ и $F(Y, H)$ се изоморфни.

3) Коваријантниот функтор $F: \mathbf{T g h} \rightarrow \mathbf{F g h}$ е инваријанта на хомотопски тип.

Head, Department of Mathematics,, Berhampore Girl's College,, BerhamPore(West Bengal), India; Pin 742102.

E-mail address: rana_pravanjan@yahoo.co.in 